J. MED. MICROBIOL. - VOL. 16 (1983) 477.482

(C) 1983 The Pathological Society of Great Britain and Ireland

\title{
SCANNING ELECTRON MICROSCOPY OF THE INTERACTION BETWEEN HAEMOPHILUS INFLUENZAE AND ORGAN CULTURES OF RAT TRACHEA
}

\author{
A. P. Johnson, J. B. ClatK* and M. F. OSBorn \\ Division of Communicable Diseases and *Electron Microscopy Research Group, M.R.C. \\ Clinical Research Centre, Watford Road, Harrow, Middlesex HA1 3UJ \\ SUMmaRY. Organ cultures of rat trachea inoculated with either a type \\ b or a non-capsulated strain of Haemophilus influenzae showed loss of \\ ciliary activity and disruption of the mucosal surface. Examination of \\ tissue pieces by scanning electronmicroscopy showed that mucosal \\ damage was due to the sloughing of epithelial cells. Bacteria associated \\ with the epithelial surface were seen infrequently and this, together with \\ the observation that sloughed cells were usually free of adherent \\ bacteria, indicated that bacterial attachment was not a necessary \\ prerequisite for the production of tissue damage.
}

\section{INTRODUCTION}

Haemophilus influenzae is an important human pathogen that causes exacerbations of chronic bronchitis and bronchiectasis in adults and bacteraemic infections, which may result in metastatic disease in the meninges or joints, in children (Turk, 1982). Organisms carried in the nasopharynx may also spread contiguously to infect the sinuses or middle ear. Virtually all strains of $\boldsymbol{H}$. influenzae that produce systemic disease are capsulated, the majority of such strains belonging to serotype $b$, whereas strains associated with bronchitis, sinusitis, otitis or conjunctivitis are generally non-capsulated (Turk, 1982).

Despite the importance of $H$. influenzae in human disease, the pathogenesis of infection either in the respiratory tract or at other sites is incompletely understood. Denny (1974) showed that either viable $H$. influenzae organisms or sterile supernatant fluids from infected cultures caused ciliostasis and epithelial damage in organ cultures of respiratory tract mucosa, and he speculated that such activity might be important in the pathogenesis of disease and might retard the clearance of bacteria from the respiratory tract. In the present study, the interaction between $H$. influenzae and organ cultures of trachea has been investigated further by scanning electron microscopy.

\section{MATERIALS AND METHODS}

Haemophilus influenzae. A type b capsulated strain of $H$. influenzae (kindly provided by Dr. C.S.F. Easmon, St. Mary's Hospital Medical School, London), and a non-capsulated strain 
(kindly provided by Dr D.C. Turk, Public Health Laboratory, Sheffield) were used. Both strains produce $\beta$-lactamase. Stock pools of organisms were stored at $-70^{\circ} \mathrm{C}$; they were thawed and cultured on chocolate agar as required. Inocula were prepared with organisms cultured overnight at $37^{\circ} \mathrm{C}$; the resulting growth was scraped from the surface of the agar with a bacteriological loop and suspended in Eagle's minimal essential medium (MEM) buffered at $p \mathrm{H}$ 7.2 with $50 \mathrm{mM}$ HEPES. This medium was prepared by diluting a concentrated stock medium (Wellcome Reagents Ltd) tenfold with sterile pyrogen-free water (Travenol Laboratories Ltd) as described previously (McGee, Johnson and Taylor-Robinson, 1976).

Organ cultures of rat trachea. Young male and female specific-pathogen-free Sprague Dawley rats bred at the Clinical Research Centre were used. The animals were killed by injecting intraperitoneally 1-2 ml of sodium pentabarbitone (Sagatal; May and Baker Ltd) and then exsanguinating them by cardiac puncture. The trachea of each animal was removed aseptically and placed in a petri-dish containing Eagle's MEM supplemented with ampicillin 10 $\mu \mathrm{g} / \mathrm{ml}$. Each trachea was cut into a series of rings which were placed individually in tissue culture tubes on a roller drum (Cherry and Taylor-Robinson, 1970); each tube contained $1 \mathrm{ml}$ of medium supplemented with ampicillin.

Inoculation of organ culture. Organ cultures were incubated on a roller drum at $37^{\circ} \mathrm{C}$ overnight and inspected to determine whether or not the ciliated mucosal cells were still active (see below). The medium was then removed from each tube and replaced with either $1 \mathrm{ml}$ of a bacterial suspension or $1 \mathrm{ml}$ of antibiotic-free medium.

Titration of bacteria in organ cultures. When tracheal rings were to be fixed for histology or scanning electronmicroscopy, the medium was removed and diluted in ten-fold steps in Eagle's MEM, and 10- $\mu$ l volumes were inoculated on to chocolate agar. The number of colonies produced after incubation for $24 \mathrm{~h}$ at $37^{\circ} \mathrm{C}$ was recorded.

Ciliary activity. The percentage of the mucosal surface of each tracheal ring with actively beating cilia was determined by direct microscopic examination as described by Cherry and Taylor-Robinson (1970). To increase the objectivity of the visual assessment of ciliary activity, the organ cultures were coded before microscopic examination.

Histology. Tissue pieces were washed gently in phosphate-buffered saline (PBS), then fixed in formol saline. They were subsequently processed by routine histological methods and sections were stained with haematoxylin and eosin.

Scanning electronmicroscopy. Tissue pieces washed gently with PBS were fixed in glutaraldehyde $3 \%$ at $4{ }^{\circ} \mathrm{C}$ overnight, washed in $0.1 \mathrm{M}$ cacodylate buffer $(p \mathrm{H} 7 \cdot 3)$ and post-fixed with osmium tetroxide $1 \%$ in cacodylate buffer. After washing again in buffer, the tissues were immersed in tannic acid $2 \%$ in cacodylate buffer for $10 \mathrm{~min}$, washed in water and fixed again in osmium tetroxide $1 \%$ in water. They were then dehydrated by passage through alcohol of increasing concentration $\left(25 \%\right.$ to $100 \%$ ) and dried at the critical point of liquid $\mathrm{CO}_{2}$ in a Polaron E3000 critical point drying apparatus. The dried tissues were mounted on aluminium stubs, coated with a thin layer of gold by a Polaron-E5100 sputter-coating apparatus and examined in a Phillips SEM500 scanning electronmicroscope.

\section{RESULTS}

In an initial series of experiments the observations reported by Denny (1974) that $H$. influenzae caused loss of ciliary activity in organ cultures of rat trachea were reproduced regularly. Organ cultures inoculated with $(1 \cdot 3-3 \cdot 5) \times 10^{8} \mathrm{cfu}$ of either a type $b$ or a non-capsulated strain showed a marked decrease in ciliary beating; frequently, little or no activity remained after incubation for 3 days. Histological examination of infected organ cultures revealed disruption of the mucosal epithelium. In contrast, control organ cultures that had not been inoculated with bacteria maintained ciliary activity; usually, $>80 \%$ of the mucosal surface had active ciliary movement 3 days after inoculation. Histological examination of control organ cultures showed that the integrity of the mucosa was generally well maintained, although small numbers of epithelial cells that had sloughed off were seen occasionally. 


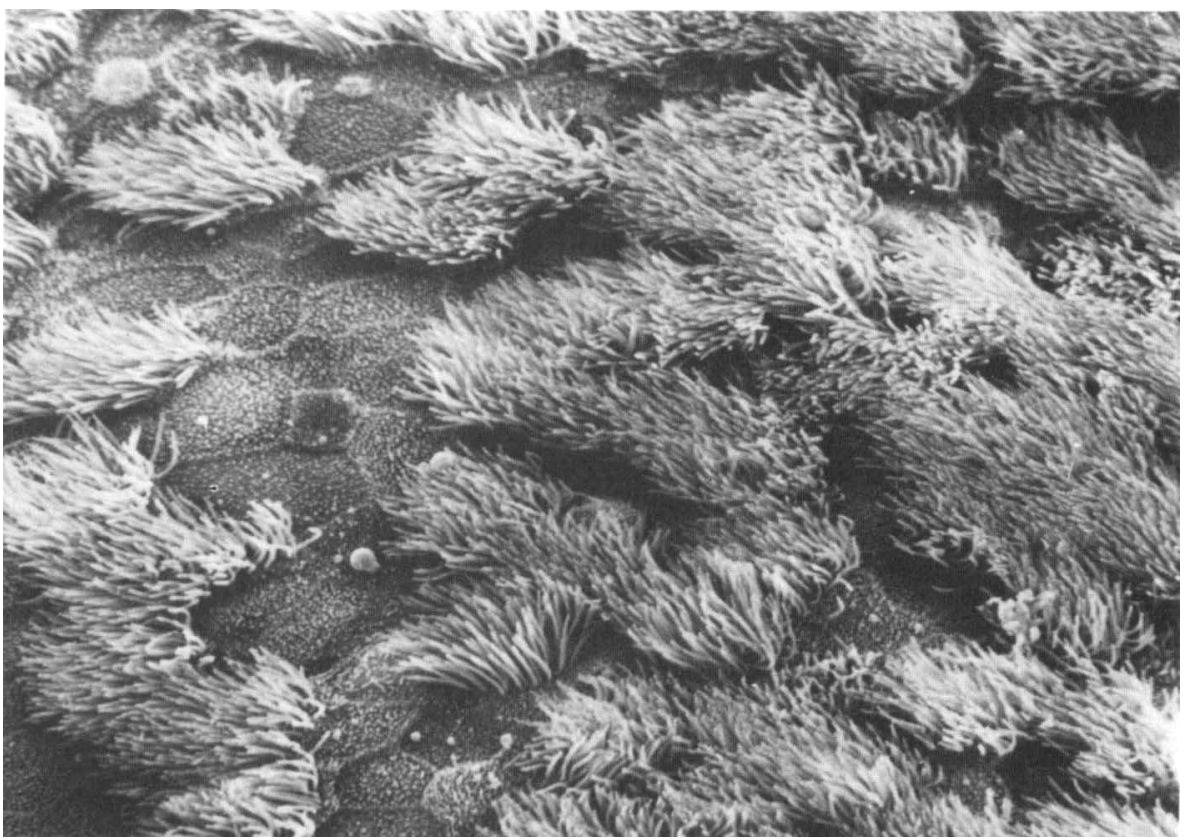

FIG. 1.-Mucosal surface of uninfected organ culture of rat trachea 3 days after preparation, showing ciliated and non-ciliated epithelial cells $(\times 2190)$.

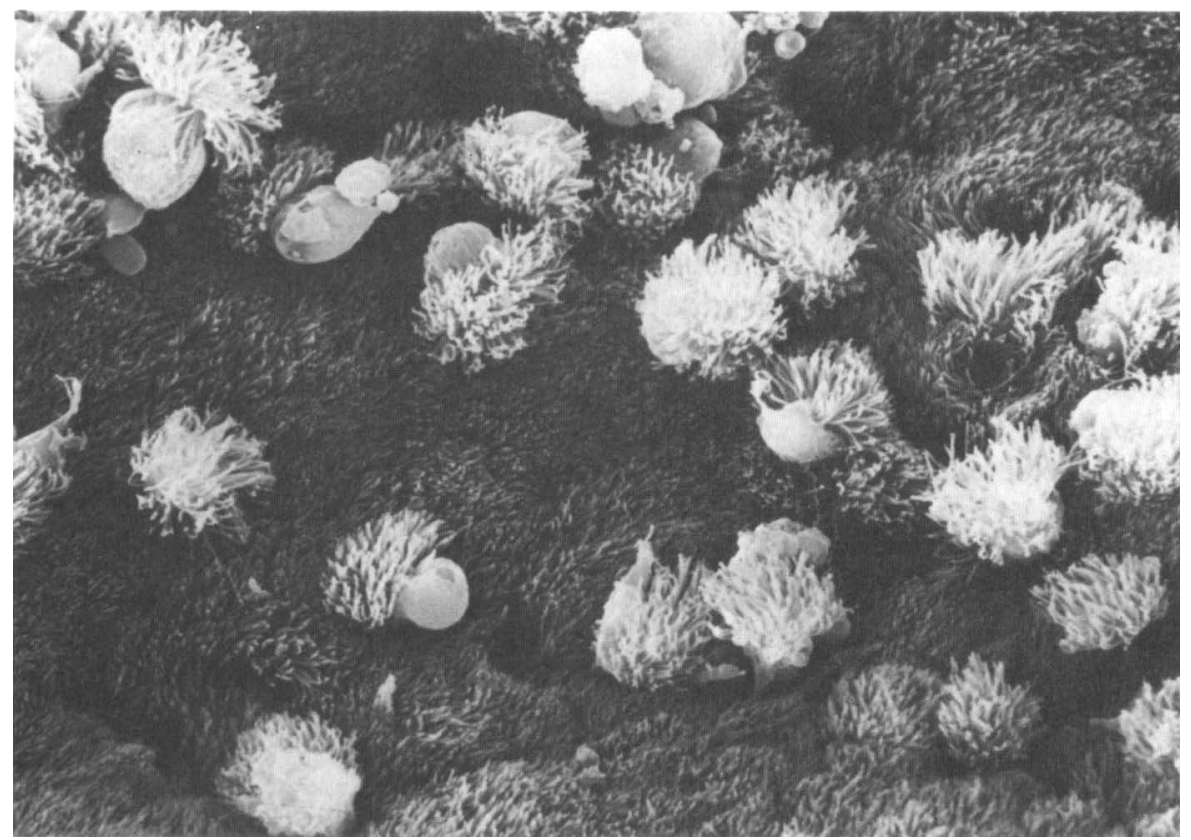

FIG. 2.-Mucosa of rat trachea 3 days after inoculation with $H$. influenzae; many ciliated cells with intact cilia have sloughed from the epithelium $(\times 2190)$. 


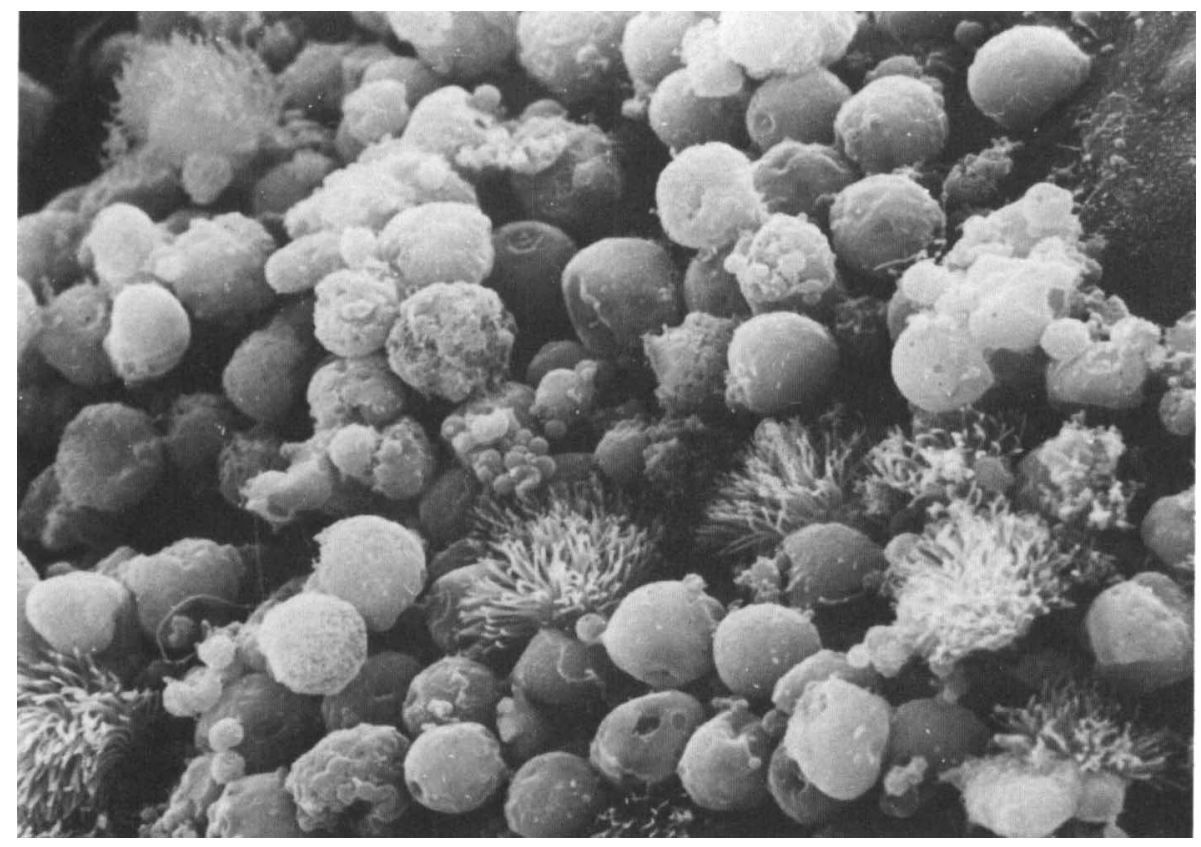

FIG. 3.-Mucosa of rat trachea 2 days after inoculation with $H$. influenzae; there is marked disruption of the epithelium with sloughing of non-ciliated cells $(\times 2190)$.

Organ cultures were examined by scanning electron microscopy to delineate further the mechanism by which loss of ciliary activity and tissue damage occurred. The mucosal surface of uninoculated organ cultures generally appeared intact (fig. 1), although occasionally a few sloughed cells were observed. The mucosa consisted of both ciliated and non-ciliated areas and the boundaries between adjacent non-ciliated cells were easily discernible (fig. 1). Organ cultures infected with either type b or non-capsulated organisms showed marked mucosal disruption with sloughing of epithelial cells (figs. 2, 3 and 4). In some areas where the mucosal surface appeared highly ciliated, the sloughed cells were predominantly ciliated (fig. 2). The presence of intact cilia on these cells and the absence of free cilia indicated that loss of ciliary activity was not due to loss of cilia from the cell surface. In contrast, in areas where the mucosa consisted of ciliated cells interspersed with areas of non-ciliated epithelium, many non-ciliated sloughed cells were seen (fig. 3).

Bacteria were seldom seen associated with the mucosal surface, irrespective of whether the organ cultures were infected with type b or with non-capsulated organisms. On the few occasions that they were observed, in such a situation, they were found associated either with cilia or with the microvilli of non-ciliated epithelial cells (fig. 4).

\section{Discussion}

The results presented here confirm the findings of Denny (1974) that both type $b$ and non-capsulated strains of $H$. influenzae caused a loss of ciliary activity in tracheal organ cultures. We have shown further that the mucosal damage occurs as a result of 


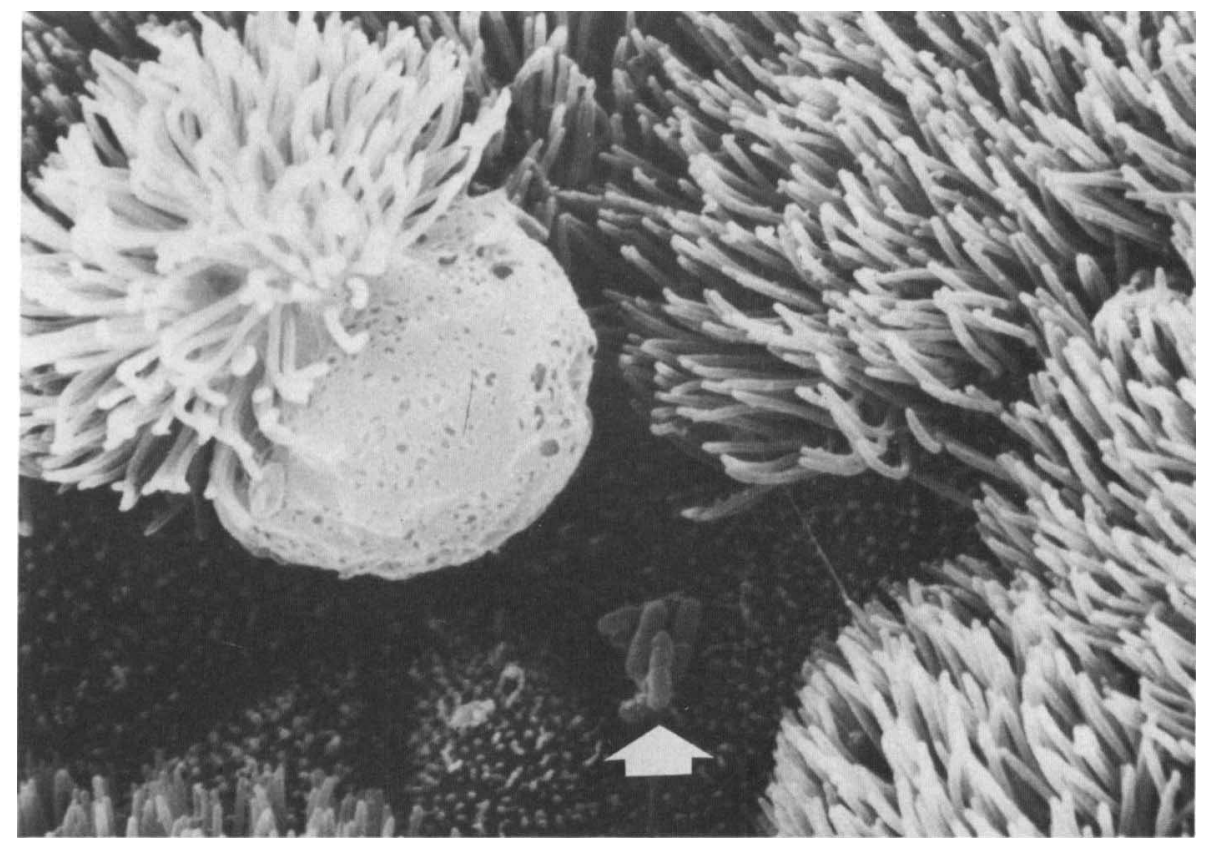

FIG. 4.-Clump of $H$. influenzae (arrow) associated with microvilli of a non-ciliated epithelial cell. Note adjacent sloughed ciliated epithelial cell free of adherent bacteria $(\times 8750)$.

the extrusion of epithelial cells. Sloughed cells were observed to be free of adherent bacteria, which indicates that direct association between bacteria and cells was not necessary for sloughing to occur. These findings are compatible with the observation reported by Denny (1974) that sterile supernatant fluids from infected organ cultures contained a soluble factor that caused loss of ciliary activity when transferred to fresh organ cultures. Denny (1974) partially characterised the soluble toxic factor present in such filtrates and suggested that it might be $H$. influenzae endotoxin. It is interesting that sterile supernatant fluids from fallopian tube organ cultures infected with Neisseria gonorrhoeae similarly cause loss of ciliary activity and sloughing of epithelial cells when transferred to fresh organ cultures (Melly, Gregg and McGee, 1981; Johnson et al., 1977) and Gregg et al. (1981) have shown that the active factor in such supernatant fluids is gonoccocal lipopolysaccharide (endotoxin).

The role that $H$. influenzae-induced extrusion of epithelial cells from the respiratory mucosa may play in the pathogenesis of disease is speculative, but it does not seem unreasonable to postulate that such activity may be relevant to the exacerbations of chronic bronchitis caused by non-capsulated $H$. influenzae. The mucociliary lining of the respiratory tract is generally regarded as an important non-specific defence mechanism against infection, and loss of ciliary activity may lower resistance to infection either with $\boldsymbol{H}$. influenzae or with other organisms. Additionally, sloughing of epithelial cells with resulting mucosal damage may provide a mechanism by which organisms can cross the mucosal barrier of the respiratory tract and come into contact with the vascular system to produce bacteraemia. The observation that non-capsulated and type $b$ organisms both damage the respiratory epithelium and should, 
therefore, have the opportunity to spread, whereas usually it is type b organisms that are associated with systemic disease, implies that type $b$ organisms have additional virulence factors that promote their invasiveness. Nevertheless, whether one is concerned with localised respiratory disease or with the systemic complications of such disease, the ability of $H$. influenzae to damage the respiratory mucosa may be an important virulence factor and deserves further investigation.

\section{REFERENCES}

DeNNY, F. W. 1974. Effect of a toxin produced by Haemophilus influenzae on ciliated respiratory epithelium. Journal of Infectious Diseases, 129, 93-100.

CHERRY, J. D. AND TAYLOR-RoBINSON, D. 1970. Large-quantity production of chicken embryo tracheal organ cultures and use in virus and mycoplasma studies. Applied Microbiology, $19,658-662$.

GregG, C. R., Melly, M. A., Hellerqvist, C. G., Coniglio, J. G. and McGee, Z. A. 1981. Toxic activity of purified lipopolysaccharide of Neisseria gonorrhoeae for human fallopian tube mucosa. Journal of Infectious Diseases, 143, 432-439.

Johnson, A. P., Taylor-Robinson, D., McGee, Z. A., Melly, M. A. and Carney, F. E. 1977. Preliminary studies on the mechanisms by which Neisseria gonorrhoeae damages host tissue. FEMS Microbiology Letters, 1, 247-249.

McGee, Z. A., Johnson, A. P. AND TAYlor-Robinson, D. 1976. Human fallopian tubes in organ culture: Preparation, maintenance and quantitation of damage by pathogenic micro-organisms. Infection and Immunity, 13, 608-618.

Melly, M. A., GregG, C. R. AND McGee, Z. A. 1981. Studies of toxicity of Neisseria gonorrhoeae for human fallopian tube mucosa. Journal of Infectious Diseases, 143, 423-431.

TURK, D. C. 1982. Haemophilus influenzae, Public Health Laboratory Service Monograph Series No. 17, Her Majesty's Stationery Office, London. 